\title{
Aspectos atuais sobre a utilização da Phyllanthus niruri (quebra-pedra) no tratamento da litíase renal
}

\author{
Aspects of current use of Phyllanthus niruri (smash-stone) in the treatment of kidney \\ lithiasis
}
Aspectos sobre el uso de Phyllanthus niruri (rompe rocas) en el tratamiento de la litiasis renal

Victor Alves de Oliveira ${ }^{1-2}$, Virleny Maria Alves de Oliveira ${ }^{2}$, Thayse Wilma Nogueira de Oliveira ${ }^{3}$, Andressa Nathanna Castro Damasceno ${ }^{4}$, Claudiane Batista de Sousa1, Thaís Rodrigues Nogueira $^{2 \star}$, Thais Alves Nogueira ${ }^{1}$, Sabrina Almondes Teixeira ${ }^{2}$, Alciene Pacheco Da Silva ${ }^{2}$, Stella Regina Arcanjo Medeiros ${ }^{2-1}$, João Marcelo de Castro e Sousa ${ }^{1}$, Felipe Carneiro Cavalcante Silva ${ }^{1}$, Gilmara Peres Rodrigues ${ }^{2-1}$.

\section{RESUMO}

Objetivo: Revisar na literatura os aspectos atuais sobre a utilização da Phyllanthus niruri (quebra-pedra) no tratamento da litíase renal. Métodos: Trata-se de uma revisão integrativa, realizada por meio de levantamento bibliográfico de publicações que investigaram o uso da Phyllanthus niruri no tratamento da litíase renal, indexadas às bases de dados Pubmed, Scielo e Lilacs. Foram incluídos artigos originais, com desenho transversal, caso-controle e coorte, bem como, artigos de revisão, em inglês, português e espanhol, sem limites de ano de publicação. Resultados: Do total de 52 artigos, 17,3\% (n=9) foram incluídos para leitura integral e discussão. Os resultados disponíveis permitiram verificar os benefícios do uso da $P$. niruri no controle da urolitíase, suas ações antiespasmódicas e anti-hiperalgésicas, e seus compostos fitoquímicos envolvidos em sua ação terapêutica. Em relação a toxicidade, esta não foi observada com uso de chás e extratos, embora seja contraindicada na gestação. Sua atividade farmacológica foi positiva, assim como seu resultado clínico e mercadológico. Final considerations: Conclui-se, portanto, que a "quebra-pedra" possui potencial clínico para abordagem terapêutica da litíase renal em humanos.

Palavras-chave: Phyllanthus niruri, Litíase Renal, Potencial Clínico.

\begin{abstract}
Objective: To review in the literature the current aspects of the use of Phyllanthus niruri (rock breaker) in the treatment of renal lithiasis. Methods: This is an integrative review, conducted through a literature review of publications that investigated the use of Phyllanthus niruri in the treatment of renal lithiasis, indexed to Pubmed, Scielo and Lilacs databases. Original articles with cross-sectional, case-control and cohort design were included, as well as review articles in English, Portuguese and Spanish, with no year of publication limit. Results: Of the 52 articles, $17.3 \%(n=9)$ were included for full reading and discussion. The available results allowed us to verify the benefits of using $P$. niruri in the control of urolithiasis, its antispasmodic and antihyperalgesic actions, and its phytochemical compounds involved in its therapeutic action. Regarding toxicity, it was not observed with the use of teas and extracts, although it is contraindicated in pregnancy. Its pharmacological activity was positive, as well as its clinical and marketing results. Final considerations: It is concluded, therefore, that the "stone breaker" has clinical potential for therapeutic approach of renal lithiasis in humans.
\end{abstract}

Key words: Phyllanthus niruri, Renal Lithiasis, Clinical Potential.

\footnotetext{
1 Universidade Federal do Piauí (UFPI), Teresina, Piauí, Brasil.

2Universidade Federal do Piauí (UFPI), Picos, Piauí, Brasil. *E-mail: thaisnogueiranutri@gmail.com

3Universidade Federal de Pernambuco (UFPE), Recife, Pernambuco, Brasil.
} 


\section{RESUMEN}

Objetivo: Revisar en la literatura los aspectos actuales del uso de Phyllanthus niruri (rompe rocas) en el tratamiento de la litiasis renal. Métodos: Esta es una revisión integradora, realizada a través de una revisión de la literatura de publicaciones que investigaron el uso de Phyllanthus niruri en el tratamiento de la litiasis renal, indexada a las bases de datos Pubmed, Scielo y Lilacs. Se incluyeron artículos originales con diseño de casos, controles y cohortes, así como artículos de revisión en inglés, portugués y español, sin límite de año de publicación. Resultados: de los 52 artículos recuperados, $17.3 \%(n=9)$ se incluyeron para la lectura completa y discusión. Los resultados disponibles nos permitieron verificar los beneficios del uso de $P$. niruri en el control de la urolitiasis, sus acciones antiespasmódicas y antihiperalgésicas y sus compuestos fitoquímicos involucrados en su acción terapéutica. En cuanto a la toxicidad, no se observó con el uso de tés y extractos, aunque está contraindicado en el embarazo. Su actividad farmacológica fue positiva, así como sus resultados clínicos y de comercialización. Consideraciones finales: se concluye, por lo tanto, que el "rompe piedras" tiene un potencial clínico para el abordaje terapéutico de la litiasis renal en humanos.

Palabras clave: Phyllanthus niruri, Litiasis Renal, Potencial Clínico.

\section{INTRODUÇÃO}

A utilização de plantas medicinais é uma prática comum das populações civilizadas em todo o mundo. A Organização Mundial da Saúde (OMS) estima que cerca de $80 \%$ da população mundial depende da medicina tradicional para o tratamento básico de saúde e, quase $85 \%$ desta envolve o uso de plantas medicinais, seus extratos vegetais e princípios ativos (OMS, 2002). No Brasil, cerca de $80 \%$ da população utiliza produtos à base de plantas medicinais nos seus cuidados com a saúde, seja pelo conhecimento tradicional, ou nos sistemas oficiais de saúde, como prática de cunho científico, orientada pelos princípios e diretrizes do Sistema Único de Saúde (SUS) (RODRIGUES AG e DE SIMONI C, 2010).

Segundo o Ministério da Saúde (2001), as plantas medicinais se caracterizam pelo histórico de uso como agente terapêutico. Os tratamentos alternativos em sua maioria derivam de produtos naturais a base de plantas na sua forma natural ou porções da planta (CORDELIA O, 2012). É crescente a procura, pela população brasileira, por alternativas ao tratamento farmacológico de inúmeras doenças. A maioria das pessoas, principalmente as de maior vulnerabilidade social, não busca atendimento médico, substituindo a assistência à saúde pela auto prescrição de produtos naturais oriundos da medicina tradicional. As consequências dessa prática incluem os riscos à saúde, por toxicidade, visto que as plantas possuem um verdadeiro arsenal químico, composto por muitas moléculas potencialmente biodinâmicas (AITA AM, et al., 2009; PAIVA SR, et al., 2016).

O reino vegetal representa um enorme reservatório de moléculas farmacologicamente ativas a serem descobertas (PAIVA SR, et al., 2016). Mundialmente, estima-se que existem aproximadamente 350.000 espécies de plantas, mas somente uma pequena porcentagem foi investigada, quanto à composição fitoquímica e potencial farmacológico. No Brasil, a diversidade genética vegetal é estimada em 55 mil espécies, o que tem exigido o esforço das universidades brasileiras para conhecer e catalogar a flora nacional, principalmente as plantas utilizadas para fins terapêuticos, com o intuito de estudar seu potencial farmacológico e preservar os conhecimentos populares sobre a medicina tradicional (DICKEL ML, et al., 2007).

As plantas pertencentes ao gênero Phyllanthus (Phyllanthaceae) conhecida pela população brasileira pela terminologia "quebra-pedra" apresentam diferentes espécies que são utilizadas na medicina popular em todo território nacional e em diversos países. Uma espécie ainda pouco estudada é a Phyllanthus niruri (AITA AM, et al., 2009). Seu uso na forma de chá é obtido por infusão do material fresco ou seco, proveniente das folhas, partes aéreas ou planta inteira, sendo recomendado pela medicina alternativa para o tratamento de litíase renal (SALEEM QE, et al., 2012).

Suas principais sinonímias científicas são P. purpurascens H.B.K; P. clorophaeus Baillon; P. ellipticus buckleye; $P$. williams standley. Estudos farmacológicos e testes pré-clínicos e clínicos confirmam as 
propriedades medicinais de $P$. amarus e $P$. niruri que têm sido mencionadas na medicina tradicional (BAGALKOTKAR G, et al., 2006; PAIVA SR, et al., 2016; ROSÁRIO ACA e ALMEIDA SSMS, 2016). Estudos experimentais e clínicos têm demonstrado que $P$. niruri tem potencial para eliminação de cálculos, aparentemente sem toxicidade aguda ou crônica. Essas propriedades medicinais estão associadas a alguns dos seus constituintes ativos, como lignanas, alcalóides, taninos, terpenos e flavonóides (BAGALKOTKAR G, et al., 2006; CALIXTO JB, et al., 1998). Porém, existem resultados controversos quanto à segurança e eficácia do seu uso na prática clínica (MARQUES LC, 2010).

Em vista desta atividade farmacológica específica, o presente trabalho realizou uma revisão da literatura científica a respeito da composição fitoquímica, toxicidade, atividade farmacológica e potencial clínico mercadológico da espécie Phyllanthus niruri (quebra-pedra) no tratamento da litíase renal.

\section{MÉTODOS}

Foi realizada uma revisão da literatura, por meio de pesquisa bibliográfica nas bases de dados Pubmed, Scielo e Lilacs. As buscas foram conduzidas por meio do formulário avançado utilizando no campo "descritores de assunto" as seguintes palavras-chave em português e seus correspondentes em inglês: plantas medicinais, fitoterápicos, Phyllanthus niruri, quebra-pedra, litíase renal.

Foram encontrados 60 documentos, dos quais foram selecionados 52 artigos de revisão e originais, com desenho transversal, caso controle e coorte, que abordavam o uso da Phyllanthus niruri (quebra-pedra) no tratamento da litíase renal.

Foram incluídos artigos originais, com desenho transversal, caso-controle e coorte, bem como, artigos de revisão, em inglês, português e espanhol. Foram excluídos da pesquisa os artigos não disponíveis na íntegra, visto que esse fato dificultava e/ou impossibilitava a extração de informações relevantes para a investigação dos fatores e desfechos estudados. Não foi estabelecido limite quanto ao ano de publicação dos artigos incluídos na presente revisão.

\section{RESULTADOS e DISCUSSÃO}

Dos 52 artigos selecionados considerando os critérios de inclusão, 9 foram incluídos na tabela de resultados para discussão com abordagem da composição fitoquímica, toxicidade, atividade farmacológica, antigenotoxicidade e outros aspectos, bem como, efeitos do uso da Phyllanthus niruri (quebra-pedra) no tratamento da litíase renal.

\section{Composição Fitoquímica}

Segundo a 4aㅡ edição da Farmacopéia Brasileira na monografia Phyllanthus niruri herbae (BRASIL, 2003), a droga vegetal é constituída pelas folhas e ramos de $P$. niruri e suas subespécies $P$. niruri ssp. niruri $L$. e $P$. niruri ssp. Lathyroides (Kunth) G.L. Webster, contendo, no mínimo, 6,5\% de taninos totais e 0,15\% de ácido gálico.

Quanto à composição fitoquímica (Quadro 1), a Phyllanthus niruri destaca-se pela presença de alcaloides, monoterpenos, triterpenos, saponinas, flavonoides, taninos, lignanas, hidrolisáveis, cumarinas e polissacarídeos.

Em um estudo realizado por Nascimento JE, et al., (2008), foi observado que a espécie Phyllanthus niruri L., não possuía alcaloides ao ser comparada com outras espécies da mesma família e gênero. No entanto, Rosário ACA e Almeida SSMS (2016) ao realizarem alguns testes, constataram que foi evidente a presença de alcaloides na $P$. niruri $L$.

Assim, pode-se esclarecer que a composição fitoquímica segundo os autores citados mostraram-se diferentes, pois provavelmente as plantas foram sujeitas a influências ambientais, o que levou a ausência e/ou presença de alcaloides na espécie. 
Quadro 1 - Composição Fitoquímica da Phyllanthus niruri (Quebra-Pedra). Teresina - PI, 2016.

\begin{tabular}{|c|c|c|}
\hline COMPOSTOS & TIPO & REFERÊNCIAS \\
\hline ALCALOIDES & $\begin{array}{l}\text { 4-hidroxisecurinina } \\
\text { 4-metoxidiidronorsecurinina } \\
\text { 4-metoxitetraidrosecurinina } \\
\text { Alosecurinina } \\
\text { Diidrosecurinina } \\
\text { Epibubbialina } \\
\text { Filocrisina } \\
\text { Nirurina } \\
\text { Norsecurinina } \\
\text { Securinina } \\
\text { Securinol A } \\
\text { Securinol B } \\
\text { Tetraidrosecurinina }\end{array}$ & $\begin{array}{l}\text { Bagalkotkar G, et al. (2006) } \\
\text { Marques LC (2010) }\end{array}$ \\
\hline MONOTERPENOS & $\begin{array}{l}\text { p-cimeno } \\
\text { Limoneno }\end{array}$ & Bagalkotkar G, et al. (2006) \\
\hline TRITERPENOS & $\begin{array}{l}\text { Acetato de lupeol } \\
\text { Lupeol } \\
\text { Filanteol } \\
\text { Filantenol } \\
\text { Filantenona }\end{array}$ & $\begin{array}{l}\text { Bagalkotkar G, et al. (2006) } \\
\text { Marques LC (2010) } \\
\text { Calixto JB, et al. (1998) }\end{array}$ \\
\hline SAPONINAS & Diosgenina & Bagalkotkar G, et al. (2006) \\
\hline FLAVONOIDES & $\begin{array}{l}\text { Astragalina, } \\
\text { Canferol-4'-ramnopiranosideo } \\
\text { Eridioctiol-7-ramnopiranosideo } \\
\text { Fisetina-4-o-glicosideo } \\
\text { Flavanona-5-o-rutinosídeo } \\
\text { Galocatequina } \\
\text { Epicatequina } \\
\text { Catequina } \\
\text { Epigalocatequina } \\
\text { Epigalocatequina-3-o-galato } \\
\text { Epicatequina-3-o-galato } \\
\text { Isoquercitrina } \\
\text { Niruriflavona }\end{array}$ & $\begin{array}{l}\text { Ishimaru K, et al. (1992) } \\
\text { Bagalkotkar G, et al. (2006) } \\
\text { Marques LC (2010) }\end{array}$ \\
\hline
\end{tabular}




\section{Revista Eletrônica Acervo Saúde / Electronic Journal Collection Health ISSN 2178-2091}

\begin{tabular}{|c|c|c|}
\hline & $\begin{array}{l}\text { Nirurina } \\
\text { Quercetina } \\
\text { Quercetol } \\
\text { Quercitrina } \\
\text { Rutina }\end{array}$ & \\
\hline TANINOS & 1-O-galoil-6-O-luteoil-a-D-glicose & Ishimaru K, et al. (1992) \\
\hline LIGNANAS & $\begin{array}{l}\text { 5-demetoxinirantina } \\
\text { Diacetato de 2,3-demetoxisecoisolintetralina } \\
\text { Filantina } \\
\text { Filnirurina } \\
\text { Filtetralina } \\
\text { Hidroxinirantina } \\
\text { Hinoquinona } \\
\text { Hipofilantina } \\
\text { Isolintetralina } \\
\text { Nirantina } \\
\text { Nirfilina } \\
\text { Nirtetralina } \\
\text { Seco-4-hidroxilintetralina } \\
\text { Trimetil éter de secoisolariciresinol } \\
\text { Urinatetralina }\end{array}$ & $\begin{array}{l}\text { Bagalkotkar G, et al. (2006) } \\
\text { Calixto JB, et al. (1998) } \\
\text { Marques LC (2010) }\end{array}$ \\
\hline HIDROLISÁVEIS & $\begin{array}{l}\text { Ácido gálico } \\
\text { Ácido repandusínico } \\
\text { Corilagina } \\
\text { Geraniina } \\
\text { Nirurisideo } \\
\beta \text {-glicogalina }\end{array}$ & Bagalkotkar G, et al. (2006) \\
\hline CUMARINAS & $\begin{array}{l}\text { Ácido elágico } \\
\text { Metilbrevifolincarboxilato }\end{array}$ & Bagalkotkar G, et al. (2006) \\
\hline POLISSACARÍDEOS & $\begin{array}{l}\text { Xilana } \\
\text { Heteroxilana }\end{array}$ & Marques LC (2010) \\
\hline
\end{tabular}

Fonte: Oliveira VA, Oliveira VMA, Oliveira TWN, et al., 2016. 


\section{Toxicidade}

Muitas plantas medicinais podem ser tóxicas dependendo da dosagem em que são administradas (MARQUES LC, 2010).

Com relação à $P$. niruri, em 2006, o Programa de Pesquisas de Plantas Medicinais da Central de Medicamentos do Ministério da Saúde, publicou com base no parecer técnico no 043/85, que esta espécie é desprovida de efeito tóxico agudo (BRASIL, 2006).

Em conformidade, estudo clínico realizado por Santos DR (1990), para avaliar a ocorrência de possíveis efeitos toxicológicos agudos em 6 voluntários saudáveis, recebendo dosagem popularmente utilizada por pacientes litiásicos (chá preparado com o pó da planta, em $200 \mathrm{~mL}$ de água, ingerido pela manhã), com intervalo de uma semana entre as doses sucessivas e crescentes de 5,10 e $15 \mathrm{~g}$ do pó de $P$. niruri, não provocou alterações no exame físico, testes psicológicos, eletrocardiográficos e de parâmetros sanguíneos.

De modo semelhante, Nishiura JL, et al., (2004) ao estudarem 69 pacientes formadores de cálculos de cálcio, tratados com chá de $P$. niruri L., composto por $450 \mathrm{mg}$ de extrato aquoso e $2 \%$ liofilizado ou placebo, ingeridos três vezes ao dia, durante 3 meses (a dose diária total foi definida arbitrariamente, sendo aproximadamente 15 vezes menor que a dose empregada em estudos toxicológicos prévios), não produziu efeitos adversos ou tóxicos nos pacientes. Os dados sobre eficácia sugerem efeitos que promovem a eliminação de cálculos renais nos pacientes, bem como a normalização dos níveis de cálcio nos que apresentaram hipercalciúria.

Outro estudo controlado, realizado por Santos DR (1990), avaliou a ocorrência de toxicidade ou de modificações nos valores dos parâmetros bioquímicos quando considerado o uso continuado. Nessa intervenção, que adotou uma posologia de $28,6 \mathrm{mg}$ para cada $\mathrm{kg}$ de peso, a administração de $P$. niruri por três meses na forma de chá dissolvido em $20 \mathrm{~g}$ do pó da planta em $500 \mathrm{~mL}$ de água, não demonstrou um potencial tóxico em pacientes com comprometimento renal.

Barros ME, et al., (2006) também relataram ausência de toxicidade aguda ou crônica pelo uso do chá de $P$. niruri, e seus achados são sugestivos de normalização dos níveis de cálcio e eliminação de urólitos em pessoas hipercalciúricas.

Boim MA, et al., (2010) corroboram a ausência de toxicidade por P.niruri sobre os sistemas renal, cardiológico e neurológico em seres humanos.

Por outro lado, considerando a possibilidade de ocorrer efeitos adversos pelo uso de plantas medicinais, resultados como os de Souza GMK, et al., (2000), devem ser considerados. Estes autores, avaliaram a administração, por via intraperitoneal, de extrato aquoso (obtido à quente) de $P$. niruri nas concentrações de 0,278 e 2,53 mg $/ \mathrm{mL}$, durante 14 dias, em ratas prenhas, sacrificadas e avaliadas quanto aos níveis séricos de hormônios tireoideanos ao final do experimento. Os resultados demonstraram aumento expressivo dos hormônios T3 (145,3\%) e T4 (83,3\%), com alterações morfofisiológicas nos embriões, o que contraindica o uso da planta durante a gravidez.

Resultados como os supracitados, embora relatados na literatura em menor frequência, reforçam a necessidade de que estudos clínicos sejam realizados na perspectiva de avaliar a eficácia do uso de $P$. niruri no tratamento de urolitíases em associação aos efeitos adversos decorrentes do uso a curto e longo prazo, nos diferentes ciclos da vida (SALEEM QE, et al., 2012).

\section{Atividade Farmacológica}

Dentre os mais de 50 compostos identificados no P. niruri, os alcaloides, flavonoides, lignanas e triterpenos têm demonstrado importantes ações relacionadas ao tratamento das urolitíases. Os alcaloides presentes no gênero Phyllanthus apresentam atividade antiespasmódica, pois favorecem o relaxamento do músculo liso e a eliminação do cálculo urinário. Os triterpenos inibem a citotoxicidade induzida pelo oxalato de cálcio ( $\mathrm{CaOx})$, além de reduzir a deposição de cristais renais. Triterpenospentacíclicos foram identificados como 
hepatoprotetores, hipolipidêmicos, antilitogênicos, anticancerígenos e antiinflamatórios (CALIXTO JB, et al., 1998; BARROS ME, et al., 2006; BOIM MA, et al., 2010; COUTO AG, et al., 2013).

O lupeol, um dos triterpenos, apresenta atividade redutora da formação de cálculos pela diluição de substâncias promotoras, bem como por ação protetora dos tecidos (COUTO AG, et al., 2013).

Além da utilização do extrato aquoso de $P$. niruri, inibir a internalização de cristais de $\mathrm{CaOx}$ em células renais de cães (MDCK), sem evidência de citotoxicidade, Freitas AM, et al., (2002) demonstraram que o extrato aquoso de $P$. niruri, impediu o crescimento de cálculos, em um modelo de urolitíase induzida por $\mathrm{CaOx}$ em ratos. $O$ alcaloide phyllantimida, presente na $P$. niruri (quebra-pedra), apresenta atividade miorrelaxante e antiespasmódica comparável à papaverina (alcaloide), o que facilita a eliminação de cálculos cravados nos ureteres.

Segundo Calixto JB, et al., (1998), os extratos obtidos de folhas, caules e raízes de $P$. niruri possuem propriedades antiespasmódicas, quando avaliados em diversos modelos biológicos de musculatura lisa, entre os quais, no íleo e bexiga urinária de cobaia, no útero de ratas, ureter e musculatura lisa vascular de cachorros. Ressalta-se que o efeito antiespasmódico foi mais expressivo utilizando o extrato preparado à base de éter como solvente.

Acredita-se que a eliminação dos cálculos renais pelo uso da infusão de folhas de $P$. niruri (classificada pelos autores como $P$. sellowianus), deve-se à ação antiespasmódica (CALIXTO JB, et al., 1998).

Em outro aspecto, Bagalkotkar G, et al., (2006), numa revisão específica sobre as propriedades farmacológicas de $P$. niruri, afirmam que os efeitos antiespasmódicos podem ser atribuídos também à presença de flavonóides, como a quercetina, e alcalóides, a exemplo da norsecurinina.

Com relação aos principais efeitos farmacológicos da Phyllanthus niruri (Tabela 1), é apresentado uma síntese de alguns estudos encontrados. Efeitos antiespasmódicos, antiplasmodial, antioxidantes, antimicrobianos e outros podem ser observados.

\section{Potencial Clínico e Mercadológico}

Apesar dos inúmeros progressos substanciais na fisiopatologia e tratamento da urolitíase, são poucos os medicamentos adequados para uso na terapia clínica. Por conta disso, um fitoterápico para a prevenção e tratamento desta doença, bem como para evitar sua recorrência, seria de grande interesse para a população e para a classe médica (BARROS ME, et al., 2003).

O uso tradicional de $P$. niruri no tratamento de cálculos renais, aliado aos resultados dos ensaios farmacológicos pré-clínicos e clínicos realizados com o extrato aquoso (ou infusão) da planta, confirmam o potencial clínico na prevenção da litíase renal, e sua ação antiespasmódica e anti-hiperalgésica. Reforça-se, desta forma, o uso terapêutico da planta $P$. niruri (quebra-pedra), na forma de infuso ou de um medicamento fitoterápico, contendo o extrato aquoso da planta (CALIXTO JB, et al., 1998; BAGALKOTKAR G, et al., 2006; BARROS ME, et al., 2006; MOREIRA J, et al., 2013).

Nesse sentido, Marques LC (2010), avaliou o potencial clínico e de registro do fitoterápico produzido a base de $P$. niruri, demonstrando que o registro de um produto fitoterápico à base de "quebra-pedra" pode ser considerado promissor. Porém, é necessário ainda, que esforços sejam realizados para a complementação das informações existentes na literatura, com o intuito de padronizar o extrato, bem como para condução de estudos clínicos em parceria com a ANVISA, que determinem dosagens, duração do tratamento e possibilidades de efeitos adversos.

Além da Índia e Itália, Espanha, China e Indonésia destacam-se pelo uso da P. niruri (quebra-pedra) na medicina tradicional (BAGALKOTKAR G, et al., 2006). 
Tabela 1 - Resumo dos Principais Efeitos Farmacológicos da Phyllanthus niruri (Quebra-Pedra) encontrados na Literatura consultada. Teresina - PI, 2016.

\begin{tabular}{|c|c|c|c|c|}
\hline Autor (Ano) & Objetivos & Partes Utilizadas & $\begin{array}{c}\text { Dosagem/ } \\
\text { Principais Resultados }\end{array}$ & $\begin{array}{c}\text { Efeitos } \\
\text { Farmacológicos }\end{array}$ \\
\hline $\begin{array}{l}\text { Calixto JB, et al., } \\
\text { (1998) }\end{array}$ & $\begin{array}{c}\text { Artigo de revisão sobre a química, estudos } \\
\text { farmacológicos, bioquímicos e clínicos in vitro e } \\
\text { in vivo da P.niruri. }\end{array}$ & $\begin{array}{l}\text { Extratos de folhas, } \\
\text { caules e raízes. }\end{array}$ & $\begin{array}{l}P . \text { niruri possuem efeitos benéficos } \\
\text { no tratamento de infecções } \\
\text { genitourinárias. }\end{array}$ & $\begin{array}{l}\text { Antinociceptiva, } \\
\text { Antiespasmódica }\end{array}$ \\
\hline Tona L, et al., (2004) & $\begin{array}{l}\text { Avaliação in vitro dos extratos de } 7 \text { plantas } \\
\text { medicinais, entre elas } P \text {. niruri. }\end{array}$ & $\begin{array}{l}\text { Planta inteira } \\
\text { (extratos) }\end{array}$ & $\begin{array}{l}\text { Atividade antiplasmodial sendo de } \\
\text { (IC50<3 } \mu \mathrm{g} / \mathrm{ml}) \text {. }\end{array}$ & $\begin{array}{l}\text { Antiespasmódica, } \\
\text { Antiplasmodial }\end{array}$ \\
\hline $\begin{array}{l}\text { Ifeoma O, et al., } \\
\text { (2013) }\end{array}$ & $\begin{array}{c}\text { Investigar a atividade antiplasmodial do extrato } \\
\text { de metanol (P.niruri) e suas frações em } 60 \\
\text { camundongos. }\end{array}$ & $\begin{array}{c}\text { Partes aéreas frescas } \\
\text { (extratos) }\end{array}$ & $\begin{array}{l}100-400 \mathrm{mg} / \mathrm{kg} \text { de peso corporal } \\
\text { durante } 2 \text { semanas. }\end{array}$ & Ação Antiplasmodial \\
\hline $\begin{array}{l}\text { Amin ZA, et al., } \\
\quad(2012)\end{array}$ & $\begin{array}{l}\text { Avaliação in vitro da bioatividade do etanol e } \\
\text { extratos aquosos de P.niruri. }\end{array}$ & $\begin{array}{l}\text { Planta inteira (Extrato } \\
\text { de etanol e aquoso). }\end{array}$ & $\begin{array}{l}\text { O extrato de etanol apresentou } \\
\text { alto teor de flavonóides e o aquoso } \\
\text { alto teor de fenol. }\end{array}$ & $\begin{array}{l}\text { Antibacteriana e } \\
\text { Antioxidante. }\end{array}$ \\
\hline $\begin{array}{l}\text { Sharma M e Singh T } \\
\text { (2013) }\end{array}$ & $\begin{array}{l}\text { Avaliação das atividades antimicrobianas do } \\
\text { extrato de etanol de diferentes partes da planta. }\end{array}$ & $\begin{array}{l}\text { Folhas frescas, } \\
\text { caules, sementes e } \\
\text { raízes (extratos) }\end{array}$ & $\begin{array}{l}\text { Utilizados } 100 \mathrm{ml} \text { de extratos } \\
\text { vegetais. }\end{array}$ & Ação Antimicrobiana \\
\hline $\begin{array}{l}\text { Okoli CO, et al., } \\
\quad(2011)\end{array}$ & $\begin{array}{c}\text { Avaliação de extratos de metanol em partes } \\
\text { aéreas da planta, sobre atividade antidiabética. } \\
\text { Realizada em ratos diabéticos. }\end{array}$ & $\begin{array}{l}\text { Partes aéreas } \\
\text { (extratos) }\end{array}$ & $\begin{array}{c}\text { O extrato reduziu a glicemia e } \\
\text { inibiu as atividades de } \alpha \text {-amilase e } \\
\alpha \text {-glucosidase }\end{array}$ & Hipoglicemiante \\
\hline $\begin{array}{c}\text { Kanchana Ke } \\
\text { Parameswari CS, } \\
(2013) \\
\end{array}$ & $\begin{array}{l}\text { Investigar o potencial nefroprotetor do extrato } \\
\text { aquoso de } P \text {. niruri contra alterações induzidas } \\
\text { pela ciclosporina no rim de ratos Wistar. }\end{array}$ & $\begin{array}{l}\text { Planta inteira } \\
\text { (extratos) }\end{array}$ & $\begin{array}{l}\text { Extrato aquoso de }(200 \mathrm{mg} / \mathrm{kg} / \mathrm{b} . \mathrm{w}) \\
\text { durante um período de } 21 \text { dias. }\end{array}$ & Ação nefroprotetora \\
\hline $\begin{array}{l}\text { De Queiroz FM, et } \\
\text { al., (2013) }\end{array}$ & $\begin{array}{l}\text { Avaliação das atividades genotóxicas, } \\
\text { antigenotóxicas e citotóxicas de um extrato } \\
\text { padronizado dessa espécie em } 48 \text { ratos } 48 \text {. }\end{array}$ & $\begin{array}{l}\text { Extrato seco de } P \text {. } \\
\text { niruri obtido em } \\
\quad \text { farmácia. }\end{array}$ & $\begin{array}{c}\text { Gavagem com } 50,150 \text { ou } \\
250 \mathrm{mg} / \mathrm{kg} / \mathrm{dia} \text {, durante } 30 \text { dias. }\end{array}$ & Antigenotoxicidade \\
\hline $\begin{array}{l}\text { Moreira J, et al., } \\
\quad(2013)\end{array}$ & $\begin{array}{l}\text { Avaliação da atividade anti-hiperalgésica de um } \\
\text { tanino isolado da } P \text {. niruri. }\end{array}$ & Planta inteira (extrato) & $\begin{array}{c}\text { Injeção intraperitoneal (1, 3, } 6 \text { e } \\
10 \mathrm{mg} / \mathrm{kg}) \text { ou gavagem }(50 \mathrm{mg} / \mathrm{kg}) \text {. }\end{array}$ & Anti-hiperalgésica \\
\hline
\end{tabular}

Fonte: Oliveira VA, Oliveira VMA, Oliveira TWN, et al, Teresina - PI, 2016. 


\section{CONSIDERAÇÕES FINAIS}

Os achados discutidos nessa revisão demonstram que o chá e extrato das folhas, raízes e caules da Phyllanthus niruri (quebra-pedra), são potenciais fitoterápicos e, portanto, estão associados à prevenção e tratamento da calculose renal. Não somente a sua composição fitoquímica, como também a diversidade de compostos envolvidos, são responsáveis por sua ação terapêutica. São poucos os estudos que fundamentam sua toxicidade, e os efeitos farmacológicos mostram-se variáveis, não sendo específicos para litíase renal. Apesar disso, o seu potencial clínico e mercadológico, confirmam os seus benefícios na prevenção de litíase renal e suas ações antiespasmódica e anti-hiperalgésica.

\section{REFERÊNCIAS}

1. AITA AM, et al. Espécies medicinais comercializadas como "quebra-pedras" em Porto Alegre, Rio Grande do Sul, Brasil. Rev. bras. Farmacogn, 2009; 19(2): 471-477.

2. AMIN ZA, et al. Assessment of in vitro antioxidante, antibacterial and imune activation potentials of aqueous and etanol exctracts of Phyllanthus niruri. Journal of the Science Food and Agriculture, 2012, 92: 1874-1877.

3. BAGALKOTKAR G, et al. Phytochemicals from Phyllanthus niruri Linn. and their pharmacological properties: areview. Journal of Pharmacy and Pharmacology, 2006; 58: 1559-1570.

4. BARROS ME, et al. Effect of extract of Phyllanthus niruri on crystal deposition in experimental urolithiasis. Urological Research, 2006; 34: 351-357.

5. BARROS ME, et al. Effects of an aqueous extract from Phyllanthus nirurion calcium oxalate crystallization in vitro. Urological Research, 2003; 30(6): 374-397.

6. BOIM MA, et al. Phyllanthus niruri as a promising alternative treatment for nephrolithiasis. International Brazilian Journal of Urology, 2010, 36(6): 657-664.

7. BRASIL. A fitoterapia no SUS e o programa de pesquisas de plantas medicinais da Central de Medicamentos. Ministério da Saúde, Brasília, 2006.

8. BRASIL. Farmacopéia Brasileira. 4ํㅡㄹ ed., tomo. 3, ed. Atheneu, São Paulo, 2003.

9. CALIXTO JB, et al. A review of the plants of the genus phyllanthus: their chemistry, pharmacology, and therapeutic potential. Medical Research Reviews, 1998, 18(4): 225-258.

10. CORDELIA O. Evaluation of chemical constituents of Phyllanthus Niruri. African Journal of Pharmacy and Pharmacology, 2012, 6(3): 125-128.

11. COUTO AG, et al. Antiinflammatory, antiallodynic effects and quantitative analysis of gallic acid in spray dried powders from Phyllanthus niruri leaves, stems, roots and whole plant. Revista Brasileira de Farmacognosia, 2013, 23(1): 124-131.

12. DE QUEIROZ FM, et al. Evaluation of (anti)genotoxic activities of Phyllanthus niruri $L$ in rat bone marrowusing the micronucleus test. Brazilian Journal of Pharmaceutical Sciences, 2013, 49(1).

13. DICKEL ML, et al. Plants popularly used for loosing weight purposes in Porto Alegre, South Brazil. Journal of Ethnopharmacology, 2007, 109: 60-71.

14. FREITAS AM, et al. The effect of Phyllanthus niruri on urinary inhibitors of calcium oxalate crystallization and other factors associated with renal stone formation. BJU International, 2002, 89(9): 829-834. Hassarajani SA, Mulchandani NB. Securinine type of alkaloids from Phyllanthus niruri. Indian J Chem 1990; 29(9):801-3.

15. IFEOMA O, et al. Isolation, fractionation and evaluation of the antiplasmodial properties of Phyllanthus niruri residente in its chloroform fraction. Asian Pacific Journal of Tropical Medicine, 2013, 169-175.

16. ISHIMARU K, et al. Phenolic constituents in tissue cultures of Phyllanthus niruri. Phytochemistryv, 1992, 31(6): 20152018.

17. JÚNIOR RFA, et al. Anti-inflammatory and antinociceptive activities of Phyllanthus niruri spray-dried standardized extract. Brazilian Journal of Pharmacognosy, 2013, 23(1): 138-144.

18. KANCHANA K, PARAMESWARI CS. Protective effect of Phyllanthus niruri again stcyclosporine A-Induced nephrotoxicity in rats.Comparative Clinical Pathology, 2013, 22(5): 885-893.

19. LIN WC, et al. Protective effect of Flos carthami extract against ethylene glycol-induced urolithiasis in rats. Urology Research, v.40, p.655-661, 2012.

20. LORENZI H, MATOS FJA. Plantas medicinais no Brasil - nativas e exóticas. Nova Odessa: Instituto Plantarum. 2002.

21. ALINI MM, et al. Protective effect of triterpenes on calcium oxalate crystal-induced peroxidative changes in experimental urolithiasis. Pharmacological Research, 2000, 41(4): 413-418. 
22. MARQUES LC. Phyllanthus niruri (Quebra-Pedra) as a Cure for Kidney Stones: a Proposal in Support of its Classification for Simplified Registration as a Phytomedicine. Revista Fitos, 2010, 5(3).

23. MARTINS ER, et al. Plantas medicinais. Viçosa: UFV. 1994.

24. MAZUMDER UK. In vitro lipid peroxidation inhibitory and antimicrobial activity of Phyllanthus niruri (Euphorbiaceae) extract.Iranian Journal of Pharmacology \&Therapeutics, 2013, 7(1): 67-70.

25. MINISTÉRIO DA SAÚDE. Proposta de Política Nacional de Plantas Medicinais e Medicamentos Fitoterápicos. 2001.

26. MOREIRA J, et al. Antihyperalgesic activity of corilagin, a tannin isolated from Phyllanthus niruri $L$ (Euphorbiaceae). Journal of Ethnopharmacology, 2013, 146: 318-323.

27. NASCIMENTO JE, et al. Estudo fitoquímico e bioensaio toxicológico frente a larvas de Artemia salina Leach.de três espécies medicinais do gênero Phyllanthus (Phyllan-thaceae). Revista de Ciências Farmacêutica Básica e Aplicada, 2008, 29(2): 145-150.

28. NISHIURA JL, et al. Phyllanthus niruri normalizes elevated urinary calcium levels in calcium stone forming (CSF) patients. Urological Research, 2004, 32(5): 362-366.

29. OKOLI CO, et al. Studies on the possible mechanisms of antidiabetic activity of extract of aerial parts of Phyllanthus niruri. Pharmaceutical Biology, 2011, 49(3): 248-255.

30. ORGANIZACIÓN MUNDIAL DE LA SALUD (OMS). Estrategia de la OMS sobre medicina tradicional2002-2005. Genebra, Switzerland, 2002.

31. PAIVA SR, et al. Uso de Plantas Medicinais Pode Trazer Riscos à Saúde Humana? Interagir: pensando a extensão, 2016, (11): 121.

32. RODRIGUES AG, DE SIMONI C. Plantas medicinais no contexto de políticas públicas. Informe Agropecuário, Belo Horizonte, 2010, 31(255): 7-12.

33. ROSÁRIO ACA, ALMEIDA SSMS. Análise fitoquímica da espécie Phyllanthus niruri L.(quebra-pedra). Estação Científica (UNIFAP), 2016, 6(1): 35-41.

34. ROW LR et al. Crystalline constituents of Euphorbiaceae-V: New lignans from Phyllanthus niruri Linn-The constitution of phyllanthin. Tetrahedron, 1966, 22: 2899-2908, 1966.

35. SALEEM QE, et al. Clinical evaluation of herbal coded formulation urolith for treatment of urolithisis. Journal of Pharmacy and Pharmocology, 2012, 1(1): 5-9.

36. SANTOS CAM. Plantas medicinais (herbarium, flora etscientia). 2.ed. São Paulo: Ícone, p.135. 1988.

37. SANTOS DR. Chá de quebra-pedra (Phyllantus niruri) na litíase urinária em humanos e em ratos. 1990. 159p. Tese (Doutorado), Escola Paulista de Medicina, São Paulo.

38. SHARMA M, SINGH T. Antimicrobial potency of Phyllantus niruriL. on some clinical isolates. International Journal of Pharma and Bio Sciences, 2013, 4(1): 875-880.

39. SOUZA GMK, et al. Influência de Phyllanthysniruri na determinação dos hormônios tiroideanos em ratas prenhes. Em: XVI LATIN AMERICAN CONGRESSO OF PHARMACOLOGY, XXXII BRAZILIAN CONGRESS OF PHARMACOLOGY AND EXPERIMENTAL THERAPEUTICS AND VII INTERAMERICAN CONGRESS OF CLINICAL PHARMACOLOGY AND THERAPEUTICS. Associação Latino Americana de Farmacologia. Abstracts. Águas de Lindóia, 2000.

40. TONA L, et al. In vitro antiplasmodial activity of extracts and fractions from seven medicinal plants used in the Democratic Republic of Congo. Journal of Ethnopharmacology, 2004, 23: 27-32. 